\title{
Asymmetric sol-gel proton-conducting membrane
}

\author{
P. Radovanovic ${ }^{\mathrm{b}}$, M. Kellner ${ }^{\mathrm{a}}$, J. Matovic $^{\mathrm{b}}$, R. Liska ${ }^{\mathrm{a}}$ \\ ${ }^{a}$ Institute of Applied Synthetic Chemistry, TU Wien, Getreidemarkt 9/163MC; 1060 Vienna - Austria \\ ${ }^{\mathrm{b}}$ Institute of Sensor and Actuator Systems, TU Wien, Floragasse 7; 1040 Vienna - Austria
}

\begin{abstract}
Proton-conducting membranes with interpenetrating polymer network morphology have gained attention in recent years for potential replacement of standard Nafion membranes in direct methanol fuel cells. These membranes generally consist of fine interpenetrating domains of proton-conducting and mechanically-supporting polymer phases, which often leads to improvements in mechanical strength and methanol barrier properties.

Asymmetric sol-gel membranes comprising proton-conducting channels of cross-linked sulfonic acid functionalized ionomers embedded within a matrix of thermally-resistant, glassy polymer were prepared by photopolymerization starting from a polymer solution and evaluated in our laboratories. These membranes have an integral top skin layer with fine biomimetic proton-conducting channels, which provides a barrier against methanol crossover, on top of a coarser proton-conducting support. Conductivity of asymmetric membranes over a range of initial polymer concentrations and ion-exchange capacities (IEC) was just slightly lower than for the corresponding symmetric membranes. Methanol barrier properties of asymmetric sol-gel membranes were better than that of Nafion 115 membrane. The crosslinking agent functionality had a major effect on membrane conductivity. Use of trifunctional crosslinking agents resulted in significantly higher conductivities than those obtained with bifunctional agents, even surpassing the conductivity of Nafion membranes.
\end{abstract}

Keywords: fuel cell, proton exchange membrane, polymer electrolyte

\section{Introduction}

Fuel cells are increasingly used as energy sources in different fields. Fuel cells comprising proton exchange membranes are focus of active research due to their versatile applications in the automotive, stationary and portable fields. The electrolyte in fuel cells is a proton-conducting polymer membrane. A fluoro-ionomer membrane, such as Nafion available from Du Pont de Nemours, is commonly used for these applications. High price of these membranes and their limitations, such as high crossover of methanol in Direct Methanol Fuel Cells (DMFC) and performance loss under conditions of low relative humidity, led to investigations of other proton-conducting membranes from less expensive, nonfluorinated materials.

Proton-conducting membranes with interpenetrating polymer network morphology have been a subject of growing interest in recent years [1]. These materials are generally prepared by either in situ polymerization and cross-linking starting from initial reactants, or by sequential synthesis starting from a polymer network swollen with necessary precursors that subsequently react to form the interpenetrating structure within the first network. An interplay of the chemical reaction and liquid-liquid demixing kinetics has a determining effect on the final membrane morphology. Interpenetrating domains of relatively small size are typical, as opposed to macroscopic phase separation observed in most polymer blends. Such fine morphology of interpenetrating proton-conducting membranes often leads to improvement in mechanical strength and reactant barrier properties.

Preparation of asymmetric sol-gel protonconducting membrane was based on the method to make asymmetric membranes by a delayed onset of liquid-liquid demixing using the process of immersion precipitation $[1,2]$. A solution of a polymer in a solvent is cast as a thin film (sol phase) and brought into a contact with a non-solvent in a gelation bath. Solvent and non-solvent are exchanged across the polymer film I gelation bath interface creating a gradient in polymer concentration at the interface. Initially all compositions in the cast film are in the stable homogeneous region. Phase separation starts only after cast film compositions enter the phase separation region. The result of phase separation between a polymer-rich and a polymer-lean phases and the subsequent gelation of the polymer-rich phase is an asymmetric membrane with a thin skin layer, as shown in Fig. 1 [4]. The original sol-gel process was modified by incorporating acrylamide-based ionomers comprising protonconducting sulfonic groups in the polymer solution and polymerizing them before the phase separation and final gelation step. This innovative modification of the original sol-gel process led to the creation of interpenetrating proton-conductive channels within the asymmetric supporting structure serving as a mechanical support and a good barrier to the fuel cell reactants. 


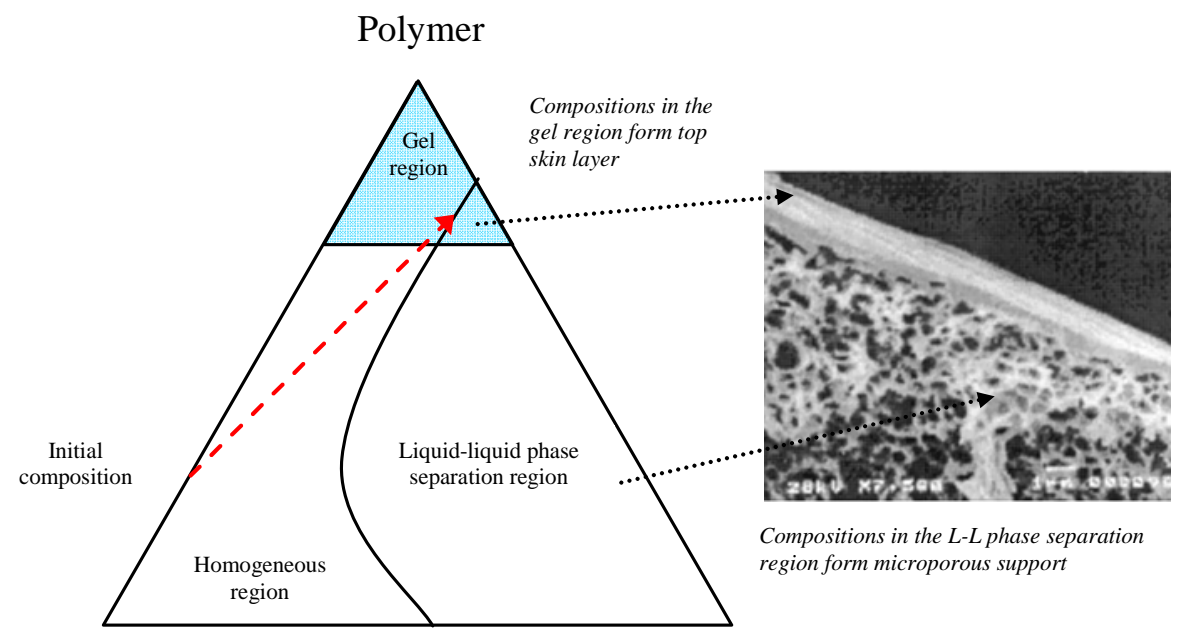

Solvent

Non-solvent

Fig. 1. Schematic ternary phase diagram of polymer solution forming asymmetric membrane by a delayed onset of liquid-liquid demixing.

\section{Experimental}

\subsection{Materials}

Polysulfone (Mw 35,000, Mn 16,000) (PSf) was obtained from Sigma-Aldrich. 2-Acrylamido-2methylpropane sulfonic acid, $99 \%$ purity (AMPS) and polyethylene glycol 700 diacrylate (PEG 700 DA) were obtained from Sigma-Aldrich. Ethoxylated (20) trimethylolpropane triacrylate (SR415) was obtained from Sartomer Company. Irgacure 2959 photoinitiator (PI) was obtained from Ciba. Polyethylene glycol 400 (PEG 400) and N-Methyl-2-pyrrolidone, $99 \%$ purity (NMP) were obtained from Fluka and Sigma-Aldrich, respectively. In all experiments deionized (DI) water was used in the final extraction bath to prepare sol-gel membranes.

\subsection{Membrane preparation}

Concentrated solutions of PSf (30 wt \%) in NMP were prepared by mixing on a hot plate overnight. Solutions of AMPS, crosslinking agent (XL) and photoinitiator in NMP were prepared fresh by mixing solutions in vials cooled with ice. Specified quantities of these solutions were mixed with a given quantity of the PSf solution in order to obtain desired compositions. Polymer concentration is expressed as weight percentage of polymer per $100 \mathrm{~g}$ of polymer and solvent (NMP). AMPS concentration is expressed in miliequivalents of sulfonic groups per $\mathrm{g}$ of final dry membrane at a theoretical $100 \%$ reactant conversion.

Prepared solutions were cast on a glass plate using a narrow film applicator with a $120 \mu \mathrm{m}$ gap (Erichsen Quadruple Applicator, Model 360) or a wide film applicator with a $127 \mu \mathrm{m}$ gap (BYK-Gardner) immersed in a PEG 400 bath, and quickly transferred into a high intensity Intelliray 600 UVB Lamp $(60 \mathrm{sec}$ exposure at $90 \%$ intensity). After UV curing, membrane samples were put into DI water to complete exchange of solvent (NMP) and non-solvent (PEG 400 ) and stored in a vial with DI water overnight to complete extraction of residuals.

Fig. 2 shows schematically the process of preparing membranes by sol-gel process used in this work. A precursor sol-gel membrane solution is cast on a glass slide and immersed into the PEG 400 bath, NMP diffuses out while PEG 400 diffuses into the cast film, shortly followed by UV exposure to start polymerization and cross-linking of AMPS - during this step it is presumed that vertical nanochannels of polyAMPS form in the top layer due to a perpendicular solvent - nonsolvent exchange across the interface (a). After polymerization, the sol-gel membrane intermediary is immersed in a water bath to extract residuals from the membrane. Final sol-gel membrane consists of a thin skin layer with biomimetic channels of cross-linked polyAMPS on top of a coarser support of interpenetrating polysulfone and polyAMPS phases (c).

\subsection{Membrane characterization}

The ion-exchange capacity (IEC) was determined by titration. Each membrane was placed in $20 \mathrm{ml}$ of $0,05 \mathrm{M}$ sodium chloride aqueous solution for $24 \mathrm{~h}$ to exchange the protons with sodium ions. The ionexchanged (hydrogen chloride) solution was titrated to $\mathrm{pH} 7.0$ with $0,01 \mathrm{M}$ sodium hydroxide aqueous solution and the end-point was detected using a phenolphthalein indicator. 


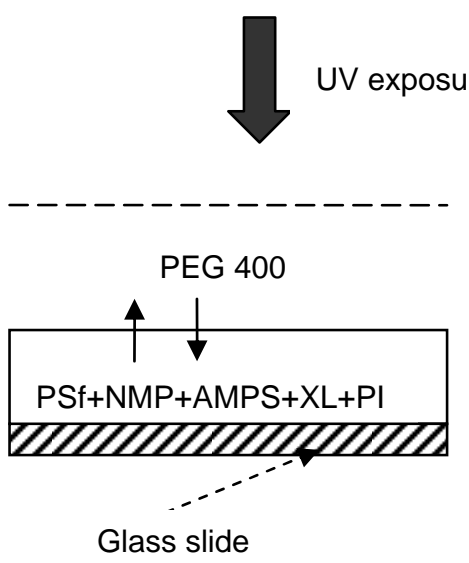

(a)

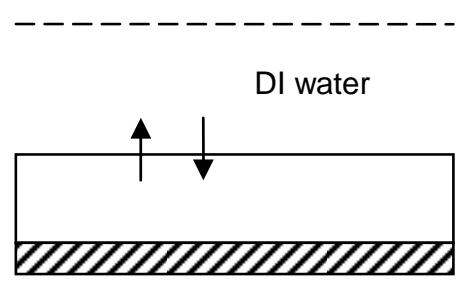

Sol-gel membrane intermediary
Biomimetic channels of cross-linked polyAMPS

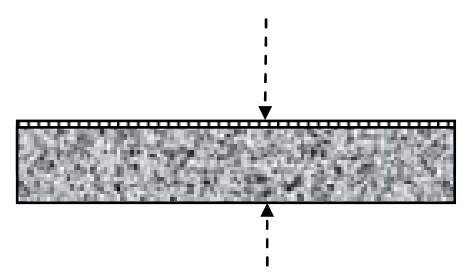

Integral microporous support of phase separated polysulfone and polyAMPS

(c)

Fig. 2. Schematic representation of the process for making sol-gel membranes.

Proton conductivity was measured in the plane of the membrane samples using a 4-point method: direct current was measured between two gold plated inner electrodes placed $1 \mathrm{~cm}$ apart, while keeping the potential difference between two gold plated outer electrodes constant at $100 \mathrm{mV}$. Membrane samples, after the extraction of reaction residues for 24 hours in DI water, were equilibrated in DI water prior to the measurements. Measured ohmic resistance and membrane thickness were then converted into electrical conductivity, which should be equal to the proton conductivity under the measuring conditions.

Methanol permeability measurements were conducted using a diaphragm diffusion cell. One compartment of the cell was filled with $50 \mathrm{ml}$ of $50 \mathrm{wt}$ \% methanol solution in water, while the other compartment contained $50 \mathrm{ml}$ of DI water. These two compartments were separated by a membrane having a $5 \mathrm{~cm}^{2}$ effective area for transport. Methanol concentration in the second compartment was monitored as a function of time by measuring refractive index of the solution. Methanol permeability was determined from the following equation [5]:

$$
P=\left(d C_{B} / d t\right)\left(V_{B} L\right) /\left(A C_{B}\right)
$$

where $d C_{B} / d t$ represents change in methanol concentration as a function of time, $V_{B}$ is the volume of the solution in second compartment, $L$ is membrane thickness, $A$ is effective membrane area, and $C_{B}$ is the average methanol concentration in the first compartment.

Scanning Electron Microscopy (SEM) images of the top and bottom surfaces of sol-gel membranes were made with FEI Quanta 200F EDAX using an environmental SEM mode at the same 20.000 magnification and 0,60 mbar pressure in order to minimize potential morphology changes due to membrane drying.

\section{Results and discussion}

Several membranes covering a range of polysulfone concentrations and initial ion exchange capacities were prepared as described in the earlier section. $10 \mathrm{~mol} \%$ of PEG 700 DA based on AMPS was used as a crosslinking agent in each of these formulations. In addition, control (symmetric) membranes were prepared using the UV curing step only (no immersion in PEG 400 to create asymmetric polysulfone concentration profile). Fig. 3 shows that the PEG 400 immersion step just slightly reduced the conductivity of sol-gel membranes. Measured in-plane conductivity of Nafion 115 was significantly higher, i.e. $0,06 \mathrm{~S} / \mathrm{cm}$. As Fig. 4 shows, IEC of sol-gel membranes measured by titration was on average only $50 \%$ of the theoretical IEC values expected based on the AMPS concentration in initial solutions. This would indicate that a significant fraction of AMPS has not been retained in the final membrane and/or was not accessible for titration. In comparison, IEC of Nafion 115 is $0,9 \mathrm{mEq} / \mathrm{g}$, which is close to the average values measured for sol-gel proton-conducting membranes.

SEM images (Fig. 5) of the top and bottom surfaces of a sol-gel membrane (made as shown in Fig. 2 from a $18 \%$ PSf solution and a 1,6 mEq/g initial AMPS concentration) reveal an asymmetric structure with cross-linked polyAMPS nanodomains of 200-300 $\mathrm{nm}$ average size on the top and much larger domains on the bottom side.

It was expected that the presence of a skin layer in such asymmetric structure would show a marked improvement in methanol barrier properties. Indeed, methanol permeability of this sol-gel membrane was an 


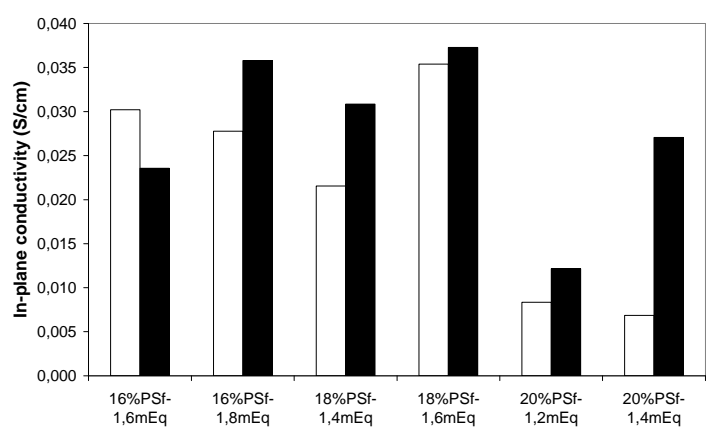

Fig. 3. In-plane conductivity of sol-gel membranes ( $\square$ ) and membranes prepared using only the UV curing step (ם).

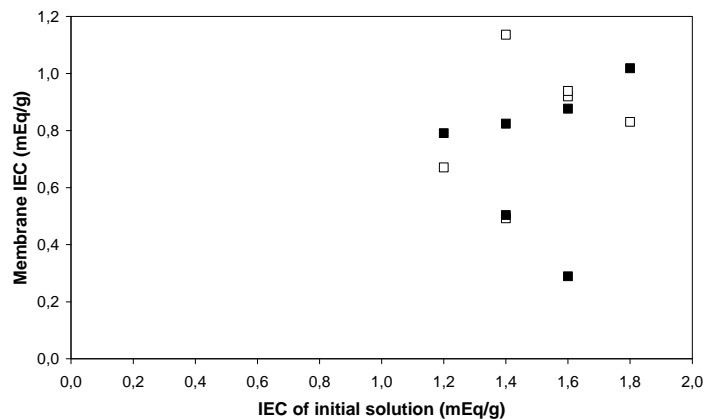

Fig. 4. Ion Exchange Capacity of sol-gel membranes ( $\square$ ) and membranes prepared using only the UV curing step

(ם).

order of magnitude lower than that of Nafion 115, i.e. $2,7 \times 10^{-7}$ vs. $2,0 \times 10^{-6} \mathrm{~cm}^{2} / \mathrm{s}$, respectively.

Relatively low measured IEC of sol-gel membranes shown in Fig. 4, led to an investigation of trifunctional crosslinking agents, which were expected be more efficient in terms of retaining a larger fraction of initial ionomer within the final membrane, compared to a bifunctional crosslinking agent, such as PEG 700 DA. As Fig. 6 shows, use of a trifunctional crosslinking agent, SR415, at $5 \mathrm{~mol} \%$ based on AMPS, resulted in significantly higher in-plane conductivities of sol-gel membranes than shown earlier in Fig. 3. Measured inplane conductivities were even higher than those of Nafion 115

\section{Conclusions}

Conductivity of asymmetric sol-gel membranes over a range of initial polymer concentrations and ionexchange capacities (IEC) was just slightly lower than for the corresponding symmetric membranes. Methanol barrier properties of an asymmetric sol-gel membrane were better than that of a Nafion 115 membrane. The crosslinking agent functionality had a major effect on membrane conductivity. Use of trifunctional crosslinking agents resulted in a significantly higher conductivity than that obtained with bifunctional agents, even surpassing the conductivity of Nafion membranes. Further optimization of these novel proton-conducting membranes and improvement in their chemical stability at higher temperatures could lead to a new generation of low cost proton-conducting membranes for DMFC.

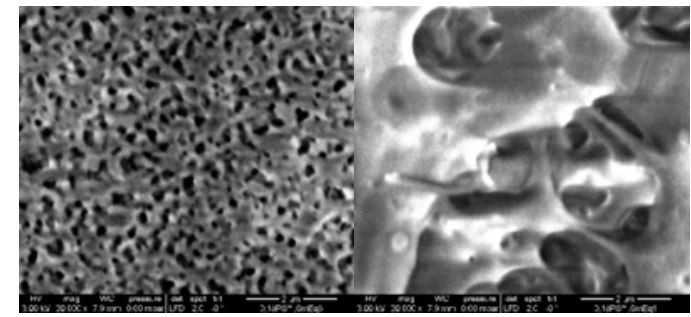

Fig. 5. ESEM images of top (left) and bottom (right) surfaces of a sol-gel membrane (bar $2 \mu \mathrm{m}$ ).

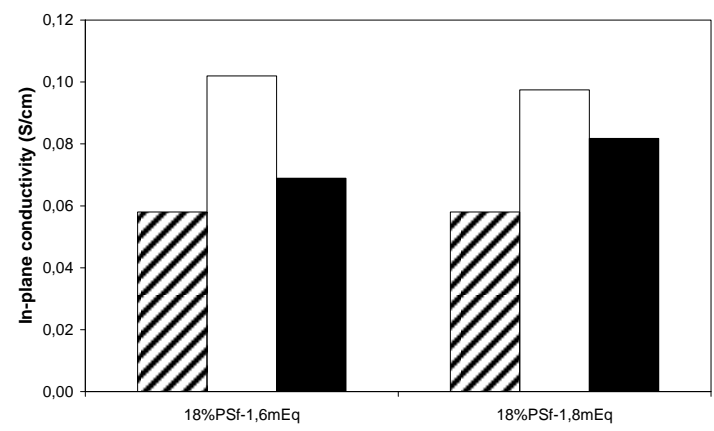

Fig. 6. In-plane conductivity of sol-gel membranes $(\square)$ and membranes prepared using only the UV curing step ( $\square)$, both made with a trifunctional cross-linking agent, compared to Nafion 115 membrane (Z).

\section{Acknowledgements}

The research leading to these results has received funding from the European Community's FP7- NMP Programme, under the Project Acronym MultiPlat and with Grant Agreement: N 228943 and the Austrian Federal Ministry of Science and Research. The authors would like to thank Adrienne Wilke for taking the SEM images and Sartomer and Ciba SC for providing monomers and initiators.

\section{References}

[1] Chikh L, Delhorbe V, and Fichet O, J. Membrane Sci. (2010), doi:10.1016/j.memsci. 2010.11.020

[2] Radovanovic $P$, Thiel SW, and Hwang ST, J. Membrane Sci. (1992), 65, 213-229.

[3] Radovanovic P, Thiel SW, and Hwang ST, J. Membrane Sci. (1992), 65, 231-246.

[4] Kim HJ, Tabe-Mohammadi A, Kumar A and Fouda AE, J. Membrane Sci. (1999), 161, 229-238.

[5] Pei H, Hong L, and Lee JY, J. Power Sources (2006) 949-956. 